\title{
Lagrangian Monte Carlo simulation of spray-flow interaction
}

\author{
T. Belmrabet ${ }^{1}$, R. Russo ${ }^{2}$, M. Mulas ${ }^{2} \& \mathrm{~S}$. Hanchi $^{1}$ \\ ${ }^{1} L M F, E M P, B P 17$ Bordj el Bahri, 16111 Alger, Algeria \\ ${ }^{2}$ Department of Computational Methods for Engineering, CRS4, CFD \\ Area, Uta (Ca), Italy
}

\begin{abstract}
The aim of this work is to determine the interaction between a droplet's stationary spray and a fluid flow, accounting for droplet vaporization, breakup and turbulences effects. In order to achieve this, a Lagrangian Monte Carlo code (McSpray) has been developed, besides a volume-finite Navier-Stokes solver (Karalis). The two codes work sequentially on the same computational grid, each influencing the other, so that a complete two-way coupling might be modelled. To validate the McSpray code in the case of two-way coupling, two cases have been performed: the first is a surface injection parallel to the inflow continuum velocity; the second is a conic point injection having an injection angle equal to 15 degrees. To verify these results, they are compared with the ones provided by Fluent commercial code.
\end{abstract}

Keywords: finite volume, spray, Monte Carlo.

\section{Introduction}

The subject of multiphase flow modelling processes is a quite vast research field of utmost practical interest. When two or more phases move relatively to each other, they may exhibit a large number of possible flow regimes. There are several classifying ways of these multiphase flows. In dispersed flows all the phases except one exist as dispersed (discontinuous) particles flowing through the continuous fluid.

Using the Eulerian-Lagrangian approach, trajectories of dispersed phase particles are simulated by solving an equation of motion for each particle. Motion of the continuous phase is modelled using a conventional Eulerian 
framework. Depending on the degree of coupling, solutions of both phases interact with each other. For two-way or four-way coupling, an iterative solution procedure needs to be adopted. In simple, one-way coupling, a continuous phase flow field can be obtained independently of the motion of the dispersed phase.

\section{Mean equation in the gas phase}

The main features of the gaseous flow are deduced from the resolution of the Reynolds Averaged Navier-Stokes (RANS) equations written for a non-reactive single fluid. The Spalart-Allmaras turbulence model is used to compute the unclosed turbulent terms.

In order to apply the numerical fully compressible formulation to incompressible flows, a preconditioning technique is used. The preconditioned system of the Navier-Stokes equations, in compact vector form, is

$$
\frac{\partial Q}{\partial t}+P\left(\frac{\partial F_{j}}{\partial x_{j}}\right)+P(\text { viscous flux })=P \cdot S
$$

where $Q$ represents the vector of conservative variables $Q=\left(\rho, \rho u_{i}, \rho E, \rho \tilde{v}\right)^{T}, F_{j}$ the corresponding inviscid fluxes and $S$ the source term vector. The preconditioning matrix is given by $P=M \cdot M_{\text {mod }}^{-1} . \boldsymbol{M}$ represents the Jacobian matrix of the vector $Q$ with respect to the vector of the so-called viscousprimitive variables $Q_{v}=\left(p, u_{i}, T, \widetilde{v}\right)^{T} . \boldsymbol{M}_{\text {mod }}$ represents a modified version of $\boldsymbol{M}$. All matrices are given in reference [1].

\subsection{Numerical method}

Equations are integrated with a cell-centered Finite-Volume method on blockstructured meshes. Convective inviscid fluxes are computed by a second order Roe's scheme [1].

In Finite-Volume and semi discrete form, the system (1) becomes

$$
\Omega \frac{\partial Q}{\partial t}=-P \cdot R E S \equiv-M \cdot M_{\text {mod }}^{-1} \cdot R E S
$$

where $\boldsymbol{R} \boldsymbol{E} \boldsymbol{S}$ represents the vector of residuals and $\Omega$ the cell volume.

Updating is done in terms of the viscous primitive variables $Q_{v}$. If an implicit numerical scheme is used to discretize the time derivative and after linearization:

$$
\left(M_{\text {mod }} \frac{\Omega}{\Delta t}+\left(\frac{\partial R E S}{\partial Q_{v}}\right)^{\text {old }}\right) \Delta Q_{v}=R E S
$$

This linear system is solved with an iterative red-black relaxation scheme [1].

\section{Representation of the dispersed phase}

\subsection{Monte Carlo technique}

Monte Carlo technique consists in calculating the characteristics of a system by generating a certain number of random events enough to catch its behaviour. 
Assuming that the evolution of the physical system can be described by a pdfs, the Monte Carlo simulation can proceed by sampling from these pdfs. This requires a fast and effective way to generate a random numbers uniformly distributed on the interval $[0,1]$. This method is used both to compute initial diameter, position and velocity of every droplet injected and to model turbulence effects by means of the "eddy interaction model".

It is well known, from experimental studies, that in general cases the particles diameters are distributed according to a so-called Rosin Rammler distribution. The initial position of the particle is determined by assuming the particles generated from an annular section with a constant density distribution over the surface. The initial velocity is computed in the same way. Currently the velocity pdfs are assumed to be Gaussian [2].

\subsection{Velocities and trajectories computing}

The Basset-Boussinesq-Oseen equation for forces balance on the droplet is [3]

$$
\begin{aligned}
& \left(1+\frac{1}{2} \frac{\rho_{c}}{\rho_{d}}\right) \frac{d v}{d t}=\frac{f}{\tau_{r}}(u-v)+\frac{3}{2} \frac{\rho_{c}}{\rho_{d}} \frac{d u}{d t}+\sqrt{\frac{9}{2 \pi}}\left(\frac{\rho_{c}}{\rho_{d}}\right)^{\frac{1}{2}} \\
& \frac{1}{\sqrt{\tau_{r}}}\left[\int_{0}^{t} \frac{\dot{u}-\dot{v}}{\sqrt{t-t^{\prime}}} d t^{\prime}+\frac{(u-v)}{\sqrt{t}}\right]+g\left(1-\frac{\rho_{c}}{\rho_{d}}\right)-\frac{v}{m_{d}} \frac{d m_{d}}{d t}
\end{aligned}
$$

where $\tau_{r}=\rho_{d} D^{2} /\left(18 \mu_{c}\right)$ and $\frac{d u}{d t}=\frac{\partial u}{\partial t}+u \cdot \nabla u$ for a stationary case.

Equation (4) is simplified neglecting the Basset force (in air $\rho_{c} / \rho_{d} \approx 10^{-3}$ ). So (4) becomes:

$$
A \frac{d v}{d t}=B(u-v)+C
$$

For a spherical particle $\mathrm{f}$ is given by a correlation proposed by Clift and Gauvin [4]. When a droplet distortion occurs f must be corrected.

Equation (5) is integrated by using a Crank-Nicholson scheme:

$$
A \frac{v^{n+1}-v^{n}}{\Delta t}=B\left(u^{*}-\frac{v^{n+1}+v^{n}}{2}\right)+C
$$

where $\mathrm{n}$ represents the time iterations number and

$$
u^{*}=\frac{1}{2}\left(u^{n}+u^{n+1}\right) \quad ; u^{n+1}=u^{n}+v^{n} \cdot \nabla u^{n} \Delta t
$$

The trajectory is obtained directly by integrating the velocity fields. During each integration step coefficient $B$ is updated to take account for evaporation and break-up.

\subsection{Break-up model}

The break-up model used in this work is the Taylor analogy break-up model (TAB) [2]. This model is suited for low Weber numbers sprays (less than 100). It is based upon an analogy between an oscillating and distorting particle and a spring mass system. 
In a non-dimensionalized form, by setting $Y=X /\left(C_{b} r\right)$, the equation of the forced oscillator becomes:

$$
\frac{d^{2} Y}{d t^{2}}=\frac{C_{F}}{C_{B}} \frac{\rho_{c}}{\rho_{d}} \frac{u^{2}}{r^{2}}-\frac{C_{k} \sigma}{\rho_{d} r^{3}} Y-\frac{C_{d} \mu_{d}}{\rho_{d} r^{2}} \frac{d Y}{d t}
$$

where $C_{B}$ is a constant equal to 0.5 . Break-up occurs for $Y>1$.

The coefficients of this equation are taken from Taylor's analogy. The numerical integration of (8) is done by considering its coefficients constant. To check whether break-up occurs, one estimates the amplitude of the oscillation, assuming no damping [2]. Anyway if break-up doesn't occur the mean $Y_{M}$ is calculated. Instead, if break-up occurs we calculate the Sauter mean diameter of the child droplets and consequently the child droplets number and velocities.

\subsection{Evaporating model}

During its trajectory each particle can evaporate or condensate according to its surrounding conditions. The rate of change of droplet mass is [3]:

$$
\frac{d m_{d}}{d t}=\operatorname{Sh} \pi D^{2} \rho_{c} D_{v} \frac{w_{A, s}-w_{A, \infty}}{D}
$$

The vapour mass fraction at the droplet surface $w_{A, s}$ can be evaluated if the droplet temperature is known. For a dilute spray $w_{A, \infty}$ can be assumed to be equal to 0 .

From (9) and by integration it is quite straightforward to obtain:

$$
D^{2}=D_{0}^{2}-\lambda t
$$

with

$$
\lambda=\frac{4 \operatorname{Sh} \rho_{c} D_{v}}{\rho_{d}}\left(w_{A, s}-w_{A, \infty}\right)
$$

This is the so-called $\mathrm{D}^{2}$-law. It is necessary to write the energy equation for the droplet to compute the energy exchanged by the droplet with the continuum.

\subsection{Turbulence effects}

To determine the droplet transport due to turbulence, the "eddy interaction model" is used [5]. This approach models the turbulence flow as a set of random eddies each characterized of a certain length $l_{e}$ and a certain lifetime $T_{e}$. The former dependent on a Eulerian flow scale 1 and the latter on the Lagrangian time scale $\tau_{l}$. So the continuum velocity at a given point is $u_{i}=U_{i}+u_{i}^{\prime}$ where the turbulent fluctuation $u_{i}^{\prime}$ is constant inside the eddy "radius" $l_{e}$. Moreover, thanks to the central limit theorem, $u_{i}^{\prime}$ will have a Gaussian pdf and the standard deviation can be taken equal to $\sqrt{2 k / 3}$. The Eulerian length scale and the Lagrangian time scale can be determined by a two-equation turbulence model. When using Spalart Allmaras turbulence model only a single turbulence quantity is solved. A second turbulence quantity must be available in order to assemble a turbulent kinetic energy and its dissipation rate [6]: 


$$
l_{e}=0.166 \frac{k^{\frac{3}{2}}}{\varepsilon} \quad ; \quad \tau_{l} \approx 0.135 \frac{k}{\varepsilon}
$$

The time a droplet takes to cross an eddy of $2 l_{e}$ long, assuming stokesian drag force, is

$$
t_{\text {cross }}=-\tau_{r} \ln \left[1-\left(\frac{2 l_{e}}{\tau_{r}|v-u|}\right)\right]
$$

The lower between $t_{\text {cross }}$ and eddy lifetime $\left(2 \tau_{l}\right)$ is the time a droplet actually interacts with the eddy. The trajectory of the droplet is calculated for a period equal to the interaction time.

\subsection{Source terms computation}

The source terms are calculated by adding to cach local cell counters the mass, the momentum and the energy exchanged by each droplet during its path. The 5 source terms are computed for each time step (and added to the scoring computed so far) as follows.

\section{Mass source term:}

$$
S_{M}=\left(M_{i}-M_{f}\right) S_{f}
$$

where $\left(M_{i}-M_{f}\right)$ is the droplet mass evaporated during the time step and $S_{f}$ is a scale factor to account the actual mass flow rate of the spray from the droplets total mass injected.

\section{Momentum source terms:}

$$
S_{P}=\left(M_{i} v_{i}-M_{f} v_{f}+M_{m} g \Delta t\right) S_{f}
$$

Where $M_{m}$ is the mean mass during the time step.

\section{Energy source term:}

$$
S_{E}=S_{f}\left[M_{i} e_{i}-M_{f} e_{f}+M_{m}(g \cdot \delta X)-\dot{Q} \Delta t+\left(M_{i}-M_{f}\right)\left(c_{p, d} T_{d}+e_{f}-e_{c}\right)\right]
$$

where $e_{i}$ and $e_{f}$ are respectively the initial and the final droplet kinetic energy, $\delta \mathrm{X}$ is the droplet displacement, and $e_{c}$ is the kinetic energy of the continuum in the current position.

During each droplet trajectory two more scorings are updated in order to estimate the droplets volume fraction and the mean droplets diameter in each cell [2].

\section{Results and discussion}

\subsection{Turbulence dispersion test}

To verify the accuracy of the code in the turbulence dispersion case in homogenous isotropic stationary turbulence (HIST), the results are compared to those provided by Graham and James [5]. The test consists in injection of a high number of droplets with zero initial velocity in a HIST flow with zero mean velocity. The mean squared displacement, for $\mathrm{t} \rightarrow \infty$, opportunely scaled, i.e. $\hat{X}_{d}^{2}=X_{d}^{2} /\left(2 u^{\prime 2} t \tau_{l}\right)$, is monitored while varying $\tau_{r} / \tau_{l}$ and $\alpha=2 u^{\prime} \tau_{l} / l_{e}$. 
For a cylindrical mesh, $X_{d}^{2}$ can be viewed either as the squared displacement along $\mathrm{z}$ or half the squared radial displacement.

For this and for all the following cases the cylindrical grid is an angular sector (angle $\pi / 32$ ) with periodic boundary conditions. The results are resumed in Table 1 .

Table 1: $\quad$ Results HIST.

\begin{tabular}{|l|c|c|c|}
\hline & $\hat{X}_{d}^{2}[5]$ & $\hat{X}_{d}^{2}$ (along z) & $\hat{X}_{d}^{2}$ radial) \\
\hline$\tau_{r} / \tau_{l}=0.01 ; \alpha=1.2$ & 0.99 & 0.99 & 0.99 \\
$\tau_{r} / \tau_{l}=10 ; \alpha=1$ & 0.98 & 0.98 & 0.98 \\
$\tau_{r} / \tau_{l}=10 ; \alpha=2$ & 0.83 & 0.78 & 0.79 \\
\hline
\end{tabular}

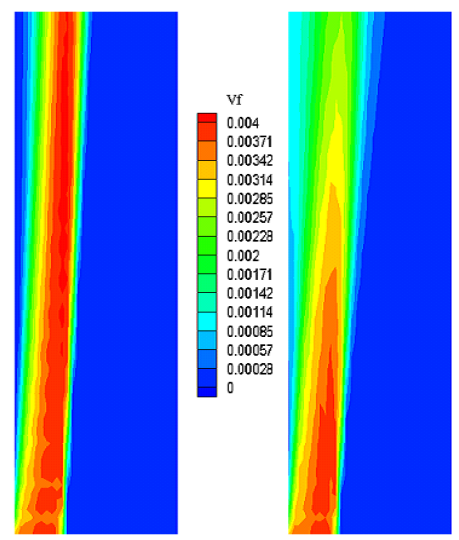

Figure 1: Volume fraction maps in laminar (left) and turbulent case (right).

\subsection{Qualitative results for one way coupling}

Now we depicted some qualitative results for a one way coupling. The effects of breakup and turbulence dispersion are also shown. In all these cases the continuum velocity is uniform and directed towards the positive $\mathrm{z}$ axis with magnitude equal to $1 \mathrm{~m} / \mathrm{s}$; the initial droplet velocity is equal to $3 \mathrm{~m} / \mathrm{s}$. The droplet size is supposed to follow a Rosin Rammler pdf, while the cone angle of the atomizer is supposed to follow a Gaussian distribution; knowing the module of velocity (assumed constant in this case) it will be possible to determine the velocity of the droplet if it is assumed no swirl at the atomizer exit.

Two test cases are performed. The first assuming no turbulence in the carrier flow and the second assuming homogenous turbulence. From Figure 1, one can notice that turbulence tends to homogenize the volume fraction distribution. For the mean droplet diameter in each cell, the influence of turbulence dispersion is shown in Figure 2. The heaviest droplets are on the spray periphery in laminar case. In turbulent case the heaviest droplets are also on the spray periphery which 
is nearly spread in all the computational volume. Figure 3 shows the effect of breakup on the energy source terms. Breakup generates smaller child particles with a slower $\tau_{r}$ which will exchange energy more rapidly.

In these cases the number of particles used is 500.000; in order to obtain source terms fields enough "smooth".

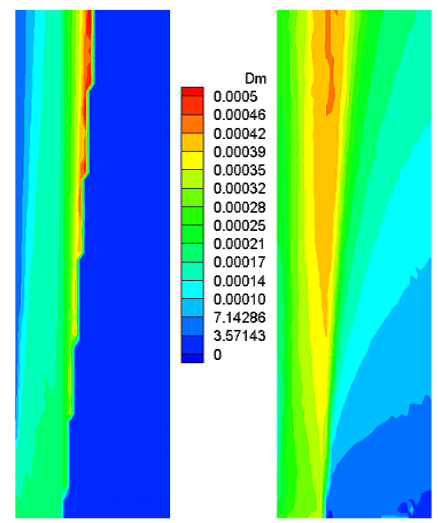

Figure 2: Mean droplet diameter maps in laminar (left) and turbulent case (right).

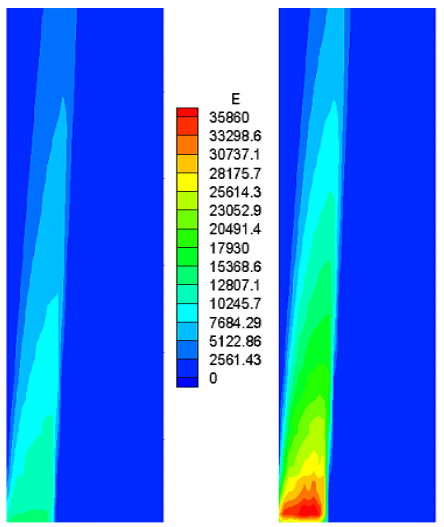

Figure 3: Energy source terms, without breakup (left) and with breakup (right).

\subsection{Qualitative results for two-way coupling}

In this case a complete coupling between continuum and water droplets in laminar case is assumed. Two cases have been performed: the first is a surface injection parallel to the inflow continuum velocity; the second is a conic point injection with injection angle equal to 15 degrees. For both of them the 
continuum inlet temperature is equal to $320 \mathrm{~K}$, and the droplets temperature is equal to $300 \mathrm{~K}$. The continuum velocity is $1 \mathrm{~m} / \mathrm{s}$ and the droplets velocity is $10 \mathrm{~m} / \mathrm{s}$. The droplet diameters are assumed to be constant.

Results are compared with the ones provided by the Fluent code (Figure 4). Here breakup model has not been used since Fluent doesn't allow breakup for stationary cases. The local and total mass and energy conservation have been verified at the end of every McSpray and Karalis run.

The number of particle used for surface injection is about $10^{6}$; a high number of particles is necessary to have a homogeneous emission from the surface (in this case we used a big area of emission, so to obtain a good statistical distribution it is necessary to use a great number of particles). For the cone injection the number of particle used is $10^{4}$, this relatively small number of particles is due to the small surface of injection (nearly a point).

\subsubsection{Surface injection}

For the first test, two continuum-spray mass flow rate ratios have been tested, respectively equal to 5 and 20 (ratio 5 and ratio 20). The results are shown in Figure 4 and Figure 5.
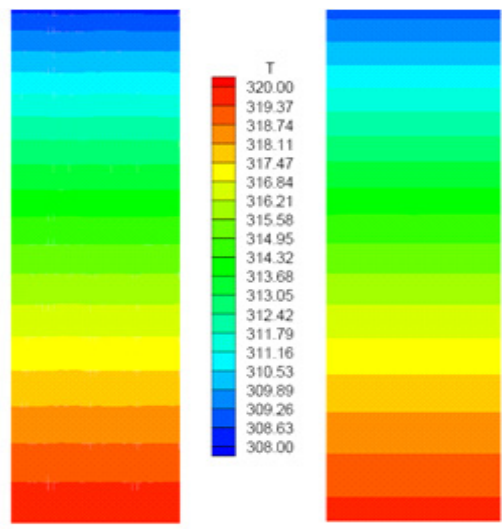

Figure 4: Continuum temperature map for ratio 5. McSpray (left) and Fluent (right).

\subsubsection{Cone injection}

For the cone injection the injection point is on the cylinder axes. The continuum/droplet mass flow rate ratio is equal to 20 . The results are shown in Figure 6.

\section{Conclusion}

The Eulerian-Lagrangian simulation of two phase flow with breakup and evaporation is performed. The effect of turbulence on spays properties (evaporation and breakup) is depicted. Good results have been found comparing 
with those provided by Graham and James [5] for HIST flow in one way coupling and by the commercial code Fluent for two-way coupling in laminar case. As future work, the McSpray code will be extended to account for wall and inter-particle collisions.

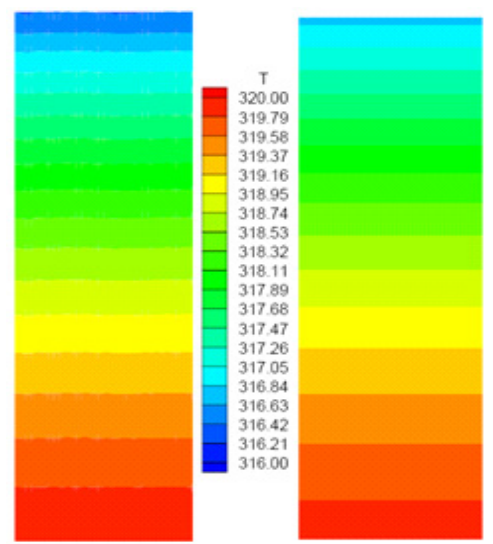

Figure 5: Continuum temperature map for ratio 20. McSpray (left) and Fluent (right).
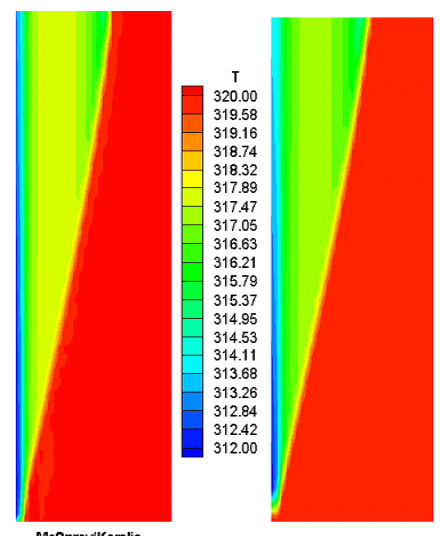

Figure 6: McSpray-Karalis results (left) and Fluent results (right).

\section{Nomenclature}

$D \quad$ particle diameter, $\mathrm{m}$

$r \quad$ particle radius, $\mathrm{m}$

$m_{d} \quad$ droplet mass, $\mathrm{kg}$

$v \quad$ droplet velocity, $\mathrm{m} . \mathrm{s}^{-1}$

$u$ continuum velocity, $\mathrm{m} . \mathrm{s}^{-1}$ 
We Weber number, $W e=\rho_{c} u^{2} r / \sigma$

$X \quad$ droplet equator displacement, $\mathrm{m}$

$Y \quad$ droplet equator displacement

$\mathrm{D}_{v} \quad$ vapor diffusion coefficient, $\mathrm{m}^{2} \cdot \mathrm{s}^{-1}$

Sh Sherwood number

$\kappa \quad$ turbulent kinetic energy $\mathrm{m}^{2} \cdot \mathrm{s}^{-2}$

\section{Greek symbols}

$\varepsilon \quad$ turbulent Dissipation, $\mathrm{m}^{2} \cdot \mathrm{s}^{-3}$

$\mu \quad$ dynamic viscosity $\mathrm{kg} \cdot \mathrm{m}^{-1} \cdot \mathrm{s}^{-1}$

$v \quad$ cinematic viscosity $\mathrm{m}^{2} \cdot \mathrm{s}^{-1}$

$\sigma \quad$ droplet surface tension N. $\mathrm{m}^{-1}$

$\tau_{r} \quad$ velocity response time, $\mathrm{s}$

Indices and exponents

$\begin{array}{ll}c & \text { continuum } \\ d & \text { droplet } \\ s & \text { vapor }\end{array}$

\section{References}

[1] Mulas, M., Chibbaro, S., Delussu, G., Di Piazza, I. \& Talice, M., Efficient parallel computations of flows of arbitrary fluids for all regimes of Reynolds, Mach and Grashof numbers, Int. J. of Numerical Methods for Heat and Fluid Flow, Vol.12 No.6, pp. 637-657, 2002.

[2] Fluent User's guide

[3] Crowe, C., Sommerfield, M. \& Tsuji, Y., Multiphase flows with droplets and particles, CRC Press LLC, ISBN 0-8493-9469-4, 1998.

[4] Clift, R. \& Gauvin, W.H., The motion of particles in turbulent gas streams, Proc. CHEMECA '70, pp. 14-28, 1970.

[5] Graham, D. I. \& James, P. W., Turbulent dispersion of particles using eddy interaction models, Int. J. Multiphase Flow Vol.22, N.1, pp. 157-175, 1996 ,

[6] Mulas, M. \& Talice, M., Fully compressible simulation of low speed premixed reactive flows, AIAA paper 4253-2003, The 33rd Fluid Dynamics Conference, (23-26 June 2003, Orlando). 